pediatric glioblastoma (GBM) DNAm data [58 female \& 91 male IDH wt samples; ages $0.1-21$ yrs; ], we found 7,371 differentially methylated cytosines (DMCs) at FDR $\leq 0.05$. Of the DMCs, 289 had DNAm differences between male and female samples $\geq 10 \%$. The majority of probes (68\%) were in CpG islands, shelves, or shores. We also found 4 differentially methylated regions (DMRs) between sexes (FWER $\leq 0.1)$. In the adult GBM DNAm samples [32 F \& $32 \mathrm{M}$ IDH wt samples; ages 22-75 yrs], we found only 117 DMCs at FDR $\leq 0.05$, and no DMRs. In the RNAseq dataset [68 F \& $54 \mathrm{M}$ pHGG samples, ages $0.08-30.6$ yrs], we found 383 differentially expressed genes (at FDR $\leq 0.05$ ), and 16 of them $(4 \%)$ overlapped a DMC. DISCUSSION/SIGNIFICANCE OF IMPACT: Our findings demonstrate that pHGG exhibits sex-specific methylome differences. Interestingly, this difference is greater in the pediatric population as compared to adults. The pHGG transcriptome also differs by sex, which may be related to differential DNAm in a minority of cases.

4485

Silicone Implant Shells Increase the Rate of Proliferation of Patient-Derived BIA-ALCL Cells but Not Primary T Cells in an Engineered Biomimetic Breast Platform

Ishani Premaratne ${ }^{1}$, Matthew Wright ${ }^{1}$, Mariam Gadjiko ${ }^{1}$, Daniel

Lara $^{1}$, Arash Samadi ${ }^{1}$, Paula Ginter ${ }^{1}$, Giorgio Inghirami ${ }^{1}$, Kristy

Brown $^{1}$, and Jason Spector ${ }^{1}$

${ }^{1}$ Clinical and Translational Science Center, Weill Cornell

OBJECTIVES/GOALS: We use a tissue engineered, biomimetic, 3D model to study the pathogenesis of breast implant-associated anaplastic large cell lymphoma (BIA-ALCL) by comparing the effect of silicone implant shell on proliferation of patient-derived BIAALCL to its precursor T cells within the breast microenvironment. METHODS/STUDY POPULATION: Patient-derived breast tissue was processed for component adipocytes, ductal organoids, and stromal vascular fraction. These were suspended within $50 \mu \mathrm{l}$ of $0.3 \%$ type I collagen matrix to which was added 200,000 cells/mL of either patient-derived BIA-ALCL cells or T progenitor cells. These were then plated into $6 \mathrm{~mm}$ wells. As a control, both BIA-ALCL cells and $\mathrm{T}$ progenitor cells were suspended within type I collagen alone at the same seeding density without breast components. Before plating, wells were lined circumferentially with either textured, smooth, or no implant shell. These were $1 \mathrm{~cm}$ by $2 \mathrm{~cm}$ pieces dissected from the whole implant. Wells were imaged using confocal microscopy over 8 days. RESULTS/ANTICIPATED RESULTS: Unstimulated T progenitor cell count showed no significant increase in any of the conditions tested. The change in cell count over 8 days was $3.85 \%$ in each condition $(\mathrm{p}=0.3352)$. A Tukey's multiple comparison test comparing each condition revealed no significant increase in cell count over 8 days for all six conditions. Notably, our previous studies have shown proliferation of BIA-ALCL cells to be significantly more robust in the biomimetic platform compared to collagen-only groups, regardless of implant shell type ( $\mathrm{p}<0.01)$. BIA-ALCL cells grew nearly $30 \%$ faster in textured and smooth shell biomimetic groups compared to biomimetic wells lacking implant shell. DISCUSSION/SIGNIFICANCE OF IMPACT: Towards elucidating BIA-ALCL's etiopathology, we show that silicone implant shell has a significant effect on proliferation of BIA-ALCL cells, but not their precursor $\mathrm{T}$ cells. If breast implant silicone shell is not a sufficient stimulus for $\mathrm{T}$ cell proliferation, co-stimulatory factors are required.
The Pain and Social Experiences Project: Understanding the role of interpersonal trauma in pain

Jennifer Pierce ${ }^{1}$, Afton L. Hassett ${ }^{1}$, Rick E. Harris ${ }^{1}$, Jenna Goesling ${ }^{1}$, and Chad M. Brummett ${ }^{1}$

${ }^{1}$ University of Michigan School of Medicine

OBJECTIVES/GOALS: Traumatic interpersonal experiences are associated with higher rates of chronic pain, increased pain severity and poorer functioning. The objective of this ongoing project is to obtain prevalence rates for various forms of interpersonal trauma among individuals with chronic pain, and to explore the potential mediating effect of heightened sensory and social sensitivity on the experience of pain. METHODS/STUDY POPULATION: Patients at Michigan Medicine between the ages of 18 and 65 complete an online survey. Patients are being recruited through a tertiary-care, outpatient pain clinic, as well as through an online health research portal. We aim to recruit 700 participants; we currently have $59.6 \%$ of our goal $(n=417)$. Participants also have the option to be included in a registry from which we can recruit for future studies. Approximately $85 \%$ of our participants have agreed to be in the registry. RESULTS/ANTICIPATED RESULTS: Preliminary data show that, of the 263 (63.4\%) participants for whom data on chronic pain is available, 167 (63.5\%) report chronic or persistent pain over the previous 3 months. Of these, $54 \%$ reported some form of childhood abuse or neglect. Approximately $41 \%$ reported four or more adverse childhood experiences. Additionally, of the 122 participants (73\%) who were in a current romantic relationship, $20 \%$ reported some form of physical violence victimization from their romantic partner. We anticipate that interpersonal trauma will be associated with poorer perceptions of social relationships, higher sensory sensitivity, and higher perceived stress. DISCUSSION/SIGNIFICANCE OF IMPACT: The PASE Project parent study will be used to better understand prevalence rates for various forms of interpersonal trauma in our chronic pain population. Future analyses and studies will explore alternative pathways linking interpersonal trauma to the experience of pain through sensory and social sensitivity, which will inform interventions aimed at reducing pain among patients with a history of trauma.

4168

\section{Understanding ECM-Based Drug Resistivity in Breast Cancer}

Sarah Libring ${ }^{1}$, Aparna Shinde ${ }^{1}$, Miad Boodaghidizaji ${ }^{1}$, Alexandra Plummer $^{1}$, Arezoo Ardekani ${ }^{1}$, Michael Wendt ${ }^{1}$, and Luis Solorio ${ }^{1}$ ${ }^{1}$ Indiana University School of Medicine

OBJECTIVES/GOALS: Cell-cell (CC) and cell-matrix interactions (CM) are known to affect drug sensitivity of cancer cells, but are not effectively recapitulated using $2 \mathrm{D}$ platforms. This research aims to determine how cell and matrix interactions confer drug resistivity in 3 distinct culturing models: 2D (no CM/limited CC), 3D spheroids (CC) and 3D fibronectin (both). METHODS/STUDY POPULATION: We examined four breast cancer cell types. The cells were derived from a nonmetastatic primary tumor (HMLE-E2) or overt bone-metastasis (BM). Transglutaminase 2 (TGM2), a matrix crosslinking protein, is overexpressed in metastatic bone tumors and may play a key role in matrix-conferred drug resistivity. In a 
gain-of-function model, TGM2 was upregulated in HMLE-E2 cells and compared to shTGM2 knockdown BM cells. Growth rates were analyzed using metabolic activity over 8 days, and drug sensitivity to Neratinib (0-1000 nM) was analyzed via cell titer. To account for the different transport properties of the 3 distinct culture environments, we developed a mathematical model for each condition, allowing us to normalize the drug sensitivity results across models to effectively compare true biological resistivity. RESULTS/ANTICIPATED RESULTS: We observed that increased cellular levels of TGM2 significantly increase the growth rate and drug resistivity of cells on fibronectin matrices. Interestingly, in 2D cultures, TGM2 expression was correlated with higher Neratinib resistivity but did not affect growth rates. In spheroid models without a significant matrix component, that rely solely on cell-cell junctions, high levels of TGM2 were correlated with lower survival rates. Lower levels of TGM2 are correlated with a more epithelial phenotype, and using our mathematical model we have identified significant transport differences between high and low TGM2 spheroids. We theorize that the low TGM2 spheroids have denser packing, which lowers the rate of diffusion and, thus reduces the effective concentration of the drug to the majority of the cells. DISCUSSION/SIGNIFICANCE OF IMPACT: Our studies indicate that the cellular response to drugs can be altered by changes in both transport properties of the tissue and the CM interactions. By systematically investigating the effects of CC interactions and CM interactions, we can use mathematical models to delineate physical means of drug resistivity from a biologically driven resistance.

\section{Regulatory Science}

\section{Implementation of Real-World Data and Real-World} Evidence in Clinical Studies

Jessica Pham ${ }^{1}$, and Eunjoo Pacifici ${ }^{1}$

${ }^{1}$ University of Southern California

OBJECTIVES/GOALS: Real-world studies have been gaining momentum in providing evidence of treatment effectiveness and hold great potential for facilitating the drug regulatory process. The U.S. Food and Drug Administration (FDA) has recognized this by providing a framework for using real-world data (RWD) to generate real-world evidence (RWE). The objective of this study is to assess the current level of RWE implementation in clinical studies. METHODS/STUDY POPULATION: Using keywords relevant to RWE, we reviewed studies on drugs, biologics, and medical devices published on PubMed in 2018. Information regarding the therapeutic area of focus, intervention type, study design, primary outcome, and data source was recorded. Further analyses of the three main therapeutic areas of study (oncology, cardiology, and infectious diseases) were performed to determine how RWE was being utilized. In addition, a broad "real-world" search was performed on Clinicaltrials.gov, from which we extracted relevant observational and Phase I, II, II/III, III, III/IV, and IV studies. A supplemental PubMed search was used to evaluate published studies in order to identify which field these trials were concentrated in and the outcome of interest.” RESULTS/ANTICIPATED RESULTS: After application of "real-world" search terms to PubMed, 995 hits were generated and of these, 311 studies were excluded. More than half of the studies were observational and retrospective in nature (64\%) with $70 \%$ examining drug/biologic outcomes. RWE data sources were largely dominated by medical records and claims data. The primary uses of RWE across oncology, cardiology, and infectious diseases included supporting drug product effectiveness, assessing safety, and evaluating treatment patterns. Of the 207 RWE studies identified on ClinicalTrials.gov, 66 were cancer randomized controlled trials (RCTs), a majority of which were used for post-marketing safety evaluations. Further research will be conducted to determine the precise role of RWE in all studies (e.g. historical comparator, label expansion). DISCUSSION/SIGNIFICANCE OF IMPACT: By examining the use of RWE in regulatory decision making, we can inform stakeholders of the extent to which robust RWE studies complement evidence generated by RCTs. Thought to reflect a product's performance in a broader and more diverse population, RWE can provide greater insight to clinical trial conduct and ultimately transform patient outcomes.

4500

Providing a System for Practical Monitoring Training for Clinical Trials within Academic Institutions

Advaita Chandramohan ${ }^{1}$, Sukhmani Kaur ${ }^{1}$, and Eunjoo Pacifici ${ }^{1}$

${ }^{1}$ University of Southern California

OBJECTIVES/GOALS: The goal was to understand the effectiveness of a novel clinical trial educational module and a corresponding initiative designed and disseminated by the Southern California Clinical and Translational Science Institute (SC-CTSI) to increase the quality of clinical trials conducted in academia. METHODS/STUDY POPULATION: The CRCs (Clinical Research Coordinators) for the initiative are asked to complete the online training. Possible study protocols are picked to be monitored by the CRCs. The monitor is instructed to study the protocol extensively and prepare for their monitoring visit. The trained monitor from the initiative then reaches out to the CRC of the study that is to be monitored and carries out the monitoring visit. Afterwards, the monitor sends initiative personnel the monitoring report, which is evaluated to see if the monitor checked everything they should have during the visit. The PI of the study is contacted with highlights from the monitoring report and improvements that they can make. RESULTS/ ANTICIPATED RESULTS: The first study monitored was a site of a large NIH-sponsored study where the consent forms were signed electronically. It was found that the monitor could not access the consent forms. Therefore, the monitor could not do source data verification. The PI of the study said that they would be raising this issue with the NIH. During the monitoring visit of the second study chosen for the initiative, patient binders were specifically examined for informed consent and source documentation completeness. The charts of patients were also reviewed. The only deviation found was a missing signature in the Investigator Site File. For the last two studies, data will be reported. DISCUSSION/SIGNIFICANCE OF IMPACT: Monitors were not only able to monitor efficiently, but also able to point out deficiencies in the monitoring practices of large studies. This model could be expanded to other academic institutions to 